\title{
Complex interactions between potentially pathogenic, opportunistic, and resident bacteria emerge during infection on a reef-building coral
}

\section{Sarah A. Gignoux-Wolfsohn ${ }^{1, *, \dagger}$, Felicia M. Aronson ${ }^{2}$ and Steven V. Vollmer ${ }^{2}$}

${ }^{1}$ Department of Ecology, Evolution, \& Natural Resources School of Environmental and Biological Sciences, Rutgers, The State University of New Jersey, New Brunswick, NJ 08901-8525, USA and ${ }^{2}$ Marine Science Center, Northeastern University, Nahant, MA 01908, USA

\author{
*Corresponding author: Department of Ecology, Evolution, \& Natural Resources School of Environmental and Biological Sciences, Rutgers, \\ The State University of New Jersey, 14 College Farm Road, New Brunswick, NJ 08901, USA. Tel: +(848) 932-9631; Fax: +(732) 932-2587; \\ E-mail: sarah.gignouxwolfsohn@rutgers.edu \\ One sentence summary: To better understand the dynamics of microbes on corals infected with white band disease, we identified potentially beneficial \\ bacteria, disease-associated foreign colonizers and disease-associated responders already present on the corals. \\ Editor: Julie Olson \\ †Sarah A. Gignoux-Wolfsohnhttp://orcid.org/0000-0002-9037-1088
}

\begin{abstract}
Increased bacterial diversity on diseased corals can obscure disease etiology and complicate our understanding of pathogenesis. To untangle microbes that may cause white band disease signs from microbes responding to disease, we inoculated healthy Acropora cervicornis corals with an infectious dose from visibly diseased corals. We sampled these dosed corals and healthy controls over time for sequencing of the bacterial $16 \mathrm{~S}$ region. Endozoicomonas were associated with healthy fragments from $4 / 10$ colonies, dominating microbiomes before dosing and decreasing over time only in corals that displayed disease signs, suggesting a role in disease resistance. We grouped disease-associated bacteria by when they increased in abundance (primary vs secondary) and whether they originated in the dose (colonizers) or the previously healthy corals (responders). We found that all primary responders increased in all dosed corals regardless of final disease state and are therefore unlikely to cause disease signs. In contrast, primary colonizers in the families Pasteurellaceae and Francisellaceae increased solely in dosed corals that ultimately displayed disease signs, and may be infectious foreign bacteria involved in the development of disease signs. Moving away from a static comparison of diseased and healthy bacterial communities, we provide a framework to identify key players in other coral diseases.
\end{abstract}

Keywords: coral disease; white band disease; staghorn coral; Acropora cervicornis; pathogenesis; symbiotic microorganisms

\section{INTRODUCTION}

Marine invertebrates are home to some of the most widely studied and complex bacterial symbioses. The deep-sea hydrothermal vent tube worms Riftia pachyptila lack mouths or guts, instead acquiring nutrients from a specialized organ containing chemoautotrophic bacteria (Cavanaugh et al. 1981). The bobtail squid, Euprymna scolopes, has also developed a specialized organ for its bacterial symbiont, Vibrio fischeri, allowing it to be bioluminescent (Nyholm and McFall-Ngai 2004). As we learn more about these mutualistic relationships, we also better understand the continuum that lies between pathogens and beneficial symbionts. Theories posit that some beneficial bacteria may have 
originally been pathogens, evolving with the host to increase host fitness (Sachs, Skophammer and Regus 2011). Recently, the genome of a sulfur-oxidizing beneficial symbiont of deep-sea mussels was found to contain homologs of toxin-encoding virulence genes, complicating our understanding of pathogens and virulence (Sayavedra et al. 2015).

The number of described marine diseases and their impacts have increased rapidly in recent years, contributing to the collapse of crucial marine ecosystems (Weil 2004; Weil and Rogers 2011; Burge et al. 2014). This increase in epizootics is likely due in part to changes in marine bacterial-animal relationships as a result of anthropogenic inputs and the changing climate. Coastal marine ecosystems and the surrounding seawater are increasingly saturated with microbes profiting from rising temperatures (Tout et al. 2015; Zaneveld et al. 2016) and increased available nutrients due to both agricultural run-off (Garren and Azam 2012) and a shift to algal-dominated ecosystems (Haas et al. 2016). This increase in microbial abundance coupled with behavioral and gene-regulatory changes in previously benign bacteria has altered definitions of disease and symbiosis. In order to understand and ultimately control these new epizootics, we need to examine how the bacterial communities associated with marine animals change, both as a cause of and in response to disease: shifting between beneficial, mutualistic and pathogenic relationships.

White band disease (WBD) is an infectious disease currently decimating populations of the two species of Caribbean Acropora coral (Acropora cervicornis and A. palmata) (Aronson and Precht 2001; Randall and van Woesik 2015). Acroporids are fast-growing reef-building corals that create habitats for numerous species of fish and invertebrates, including slower-growing species of corals (Gladfelter et al. 1977; Tunnicliffe 1983). WBD is characterized by a front of necrotic tissue (and sometimes a zone of bleached tissue), which proceeds rapidly from base to tip of the coral colony, leaving behind a band of white skeleton (Gladfelter 1982). WBD can be transmitted through the water column and by the corallivorous snail Coralliophila abbreviata (GignouxWolfsohn, Marks and Vollmer 2012). Multiple studies have confirmed that WBD signs can be caused by the bacterial fraction of a disease slurry and arrested by the administration of antibiotics, suggesting a bacterial cause of the disease (Kline and Vollmer 2011; Sweet, Croquer and Bythell 2014). Vibrio charchariae has been shown to elicit WBD signs in A. cervicornis in Puerto Rico (Gil-Agudelo, Smith and Weil 2006), and a Rickettsiales-like organism, which may be compromising the host, has been associated with both apparently healthy A. cervicornis and A. cervicornis displaying disease signs (Peters et al. 1983; Casas et al. 2004). How these and other bacteria contribute to the development of WBD signs, and whether there is a single primary WBD pathogen across the Caribbean and through time is still unknown.

Previously, Gignoux-Wolfsohn and Vollmer (2015) used 16S gene sequences to find that WBD-associated bacterial communities were significantly different from those of healthy corals. In keeping with studies of other coral diseases, we found that the bacterial communities of corals displaying disease signs were more diverse, with more consistently-associated OTUs than apparently healthy corals (e.g. Sunagawa et al. 2009; Closek et al. 2014; Roder et al. 2014a). The lack of consistent healthyassociated bacteria indicates that other factors may be shaping this microbiome. We also showed that the site of collection influenced the microbial communities as much as the disease state of the coral. The many disease-associated OTUs found among all sites became many putative WBD pathogen(s). Bacterial diseases can be caused by a few cells of a single pathogen invading the host tissues (low infectious dose) (Zwart, Daros and Elena 2011), a consortium of pathogens that may be sufficient but not necessary to cause disease signs (Lemire et al. 2015) or normally commensal bacteria reaching a threshold, which initiates a switch to pathogenic behavior (Rutherford and Bassler 2012). Furthermore, commensal bacteria could become pathogenic due to an external environmental trigger (Lesser et al. 2007). The uncertainty around which of these scenarios leads to the infectious WBD-like signs complicates our ability to determine which of the identified 'disease-associated' OTUs may be invading the host tissue and causing the disease signs and which may be responding to the necrosis, host immune response or secondary metabolites produced by the pathogen(s). We exposed corals to an infectious dose of homogenized tissue from diseased corals and sampled corals at three time points: (i) apparent health prior to exposure, (ii) apparent health post exposure, (iii) during the development of characteristic WBD signs. By using multiple coral colonies, we were able to better identify resident microbes associated with each colony, and by performing this experiment in controlled tanks we removed the possibility of an environmental trigger of pathogenicity. We examined disease-associated OTUs for consistency across two sites in order to remove the site variability we had previously found. This controlled infection experiment allowed us to answer two main questions about the diseased coral microbiome: (i) Where do these disease-associated bacteria originate? and (ii) when do they increase in abundance? We expected the final diseased-coral microbiome to be shaped by increased abundances of both bacteria originating in the dose (here referred to as colonizers) and bacteria that were found a priori on the corals (responders) increasing in abundance either before (primary) or after (secondary) development of disease signs.

\section{MATERIALS AND METHODS}

\section{Tank infection experiment}

An infection experiment was set up in July 2014, using Acropora cervicornis from two sites (CK4 and CK14) $600 \mathrm{~m}$ apart in Coral Cay, Bocas del Toro, Panama (site). At each site, corals to be inoculated were collected by taking 12 apparently healthy $5 \mathrm{~cm}$ fragments from 5 colonies (presumed to be distinct genotypes, at least $10 \mathrm{~m}$ apart) of A. cervicornis for a total of 10 colonies (colony) and corals to be made into inoculants were collected by taking three replicate $5 \mathrm{~cm}$ fragments from the disease interfaces (or equivalent location) of 3 colonies exhibiting signs of WBD and from three apparently healthy control colonies.

These fragments were brought to the Smithsonian Tropical Research Institute and the fragments to be inoculated were cable-tied to plastic louver. Ten fragments (one fragment from each colony) were placed in each of 12 closed $50 \mathrm{~L}$ tanks (tank) with a powerhead (see Fig. S1, Supporting Information, for experimental design). Corals were sampled as they were placed in tanks (time one) in the following manner: two polyps from the middle of each fragment (this small sample was used so as not to stress the coral fragment) were removed using sterile forceps and placed in $200 \mu \mathrm{l}$ of guanidine thiocyanate DNA buffer (Fukami et al. 2004). Forceps were flame sterilized in between corals. Throughout the experiment, DI water was added to maintain salinity and volume, and temperature was measured to ensure consistency across tanks.

To create the 12 inoculants (three doses and three control inoculants from each site), the three replicate fragments from 
each colony were homogenized by shaking in a falcon tube with sterile glass beads and $15 \mathrm{~mL}$ filtered seawater until no tissue remained on the skeleton (Kline and Vollmer 2011), and then pooled. Two hundred microliters of each inoculant was centrifuged and preserved in $500 \mu \mathrm{l}$ of DNA buffer.

Prior to inoculation, corals were lesioned using an airbrush and filtered seawater (Gignoux-Wolfsohn, Marks and Vollmer 2012). Six tanks were then inoculated with $30 \mathrm{~mL}$ of dose (the dose level of inoculant), three per site (inoculant site), and six tanks were inoculated with $30 \mathrm{~mL}$ of control inoculant (the control level of inoculant), three per site (inoculant site). Corals were then sampled at $10 \mathrm{~h}$ post-inoculation as described above (time two). When dosed corals began to show disease signs (i.e. the white lesion grew to encircle the coral and form the characteristic white band of skeleton) beginning at $22 \mathrm{~h}$ post-inoculation, they were sampled and removed from the experiment along with their corresponding control fragment (time three). Sampling continued in this manner until $60 \mathrm{~h}$ post-inoculation when all remaining corals were sampled (see Fig. S1 for sampling). The final disease state of a coral was determined based on whether or not that coral ultimately showed disease signs. For example, even though a sample collected at time two came from a healthy-looking coral, if that coral displayed disease signs at time three, the sample's final disease state was diseased. A total of 43 out of 60 corals that were dosed ultimately displayed disease signs. Two of the 60 control corals died over the course of the experiment and were removed from subsequent analyses (Table S1, Supporting Information).

DNA was extracted from samples using the Agencourt DNAdvance bead extraction kit (Agencourt Bioscience Corporation, Beverly, MA, USA) with the addition of PEB buffer. A blank DNA extraction was performed with each round. The V6 hypervariable region of the $16 \mathrm{~S}$ gene was chosen as the target due to its short length and high sensitivity to species-level diversity (Youssef et al. 2009; Barriuso, Valverde and Mellado 2011; Caporaso et al. 2012). The V6 region was amplified with primers consisting of a region complementary to $\mathrm{V} 6$, a unique five base pair barcode and the Illumina sequencing adapter (Gloor et al. 2010):

\section{V6-L [5'ACACTCTTTCCCTACACGACGCTCTTCCGATCTnnnnnCW ACGCGARGAACCTTACC3 '] \\ V6-R [5'CGGTCTCGGCATTCCTGCTGAACCGCTCTTCCGATCTnnn nnACRACACGAGCTGACGAC3 ' $]$}

A separate $40 \mu \mathrm{l}$ PCR reaction was performed for each sample with a unique combination of primers: $5 \mu$ l each $4 \mathrm{mM}$ primer, 8 $\mu \mathrm{l}$ standard Taq buffer (New England Biolabs, Ipswich, MA, USA), $0.8 \mu \mathrm{l}$ dNTPs, $20 \mu \mathrm{l}$ diH20, $0.5 \mu \mathrm{l}$ Taq DNA polymerase (NEB) for the following cycle: $94^{\circ} \mathrm{C}$ for $2 \mathrm{~min}$, with 28 cycles of $94^{\circ} \mathrm{C}$ for $15 \mathrm{~s}, 55^{\circ} \mathrm{C}$ for $15 \mathrm{~s}, 72^{\circ} \mathrm{C}$ for $30 \mathrm{~s}$, ending with $72^{\circ} \mathrm{C}$ for $1 \mathrm{~min}$. A negative control and blank were amplified with each set of reactions. Concentrations of PCR products were quantified using the Qubit 2.0 fluorometer (Thermo Fisher Scientific, Waltham, MA) to determine the volume of each product to pool. The pooled PCR products were then amplified with the following Illumina primers:

\section{OLJ139 [5'AATGATACGGCGACCACCGAGATCTACACTCTTTCCCTA CACGA3'] \\ OLJ140 [5'CAAGCAGAAGACGGCATACGAGATCGGTCTCGGCATTCC TGCTGAAC3 ']}

in a $40 \mu \mathrm{l}$ reaction: $8 \mu \mathrm{l}$ Phusion buffer (NEB) $0.8 \mu \mathrm{l}$ dNTPs, 0.5 $\mu \mathrm{l}$ Phusion HIfidelity Taq (NEB), $20.2 \mu \mathrm{l}$ diH20, $0.5 \mu \mathrm{l}$ DNA (previous PCR product), for the following cycle: $98^{\circ} \mathrm{C}$ for $2 \mathrm{~min}, 12 \mathrm{cy}-$ cles of $98^{\circ} \mathrm{C}$ for $1 \mathrm{~min}, 55^{\circ} \mathrm{C}$ for $1 \mathrm{~min}, 72^{\circ} \mathrm{C}$ for $1 \mathrm{~min}$ and finally $72^{\circ} \mathrm{C}$ for $5 \mathrm{~min}$. Final PCR products were cleaned using DNAmpure beads (Agencourt). Concentration and length were verified using the Agilent 2100 Bioanalyzer system (Agilent, Santa Clara, CA, USA) and sequenced using paired-end 150 base pair sequencing on an Illumina HiSeq2000.

\section{Bioinformatics}

Paired reads were overlapped using FLASH (Magoc and Salzberg 2011). Sequences were then demultiplexed, quality filtered and trimmed using a custom python script available at https://github.com/sagw/Python_scripts/blob/master/SD1/SD1_ demultiplex.py

Using Qiime 1.9.0, 97\% OTUs were picked using the open reference OTU picking method and taxonomy assigned using BLAST against the July 2015 SILVA database (Quast et al. 2013). OTUs that were identified as chloroplasts using BLAST were removed. Chimeras were detected and removed using UCHIME (Edgar et al. 2011). Further details of bioinformatics can be found at https://github.com/sagw/Notebooks/tree/master/SD1_ notebooks.

\section{Statistical analyses}

OTU counts were normalized using the sizefactors method with arithmetic means in the R package DESeq2 (Love et al. 2014; McMurdie and Holmes 2014). The significance of the community level effects was tested using PERMANOVA of Bray-Curtis dissimilarities (adonis in package Vegan) (Oksanen et al. 2013). Two PERMANOVAs were performed: one using the formula: ' colony' for time-one samples, and one using the formula: ' $\sim$ final disease state + inoculant $*$ site $*$ time $*$ inoculant site' for time-two and three samples. Site was removed from the model because the main effect and interactions were not significant. Shannon diversity and rarefied richness were calculated using Vegan (Oksanen et al. 2013).

To evaluate changes in abundance of individual OTUs among main effects and interactions with the addition of random effects, abundance data for each OTU were fit to quasipoisson mixed-effects generalized linear models (GLMMPQL in package MASS) (Venables and Ripley 2003). GLMMs for time-one samples used the fixed-effect formula: ' colony' and the random effect formula: ' 1-tank'. GLMMs for time-two and three samples used the fixed effect formula: ' $\sim$ final disease state + site $*$ inoculant $*$ time $*$ inoculant site' and the random effect formula: ' 1-tank/time'. Significance of effects was then determined by type III ANOVA using the Wald chi-square test (ANOVA in package Car) (Fox and Weisberg 2011) and significantly different OTUs $(P$-value adjusted by false discovery rate $<0.05$ ) were determined for each main effect and interaction. OTUs were then grouped according to significance of GLMM terms and post hoc calculated means, and mean abundance of a subset of OTUs was plotted using ggplot2 (Wickham 2009).

\section{OTU group definitions}

We identified colony-specific healthy residents as OTUs that differed significantly by colony at time one and by final disease state at times two and three, with a higher abundance in control than dosed diseased corals. The majority of these OTUs belonged to the genus Endozoicomonas, and so the mean of each OTU identified as Endozoicomonas was calculated for each colony and \% Endozoicomonas composition was calculated as a mean of the \% of the total microbiome for each sample belonging to a given colony. 
Table 1. PERMANOVA of Bray-Curtis dissimilarity between samples collected at times two and three.

\begin{tabular}{llcccc}
\hline Effect & $\mathrm{df}$ & Sums of Sqs & Mean Sqs & F Model & $R^{2}$ \\
\hline Final disease state & 1 & 3.49 & 3.49 & 9.43 & 0.030 \\
Inoculant & 1 & 1.06 & 1.06 & 2.86 & 0.0092 \\
Time & 1 & 7.77 & 2.59 & 7.00 & 0.067 \\
Inoculant site & 1 & 0.89 & 0.89 & 2.40 & 0.0077 \\
Inoculant x time & 1 & 1.67 & 0.83 & 2.25 & 0.014 \\
Inoculant x inoculant site & 1 & 1.20 & 1.20 & 3.23 & 0.010 \\
Time x inoculant site & 1 & 2.41 & 0.80 & 0.001 \\
Inoculant x time x inoculant site & 1 & 1.60 & 0.80 & 2.15 & 0.020 \\
Residuals & 162 & 95.46 & 0.37 & & 0.014 \\
Total & 178 & 115.53 & & & 0.001 \\
& & & & & 0.001 \\
\end{tabular}

We identified bacteria that are likely involved in the etiology of the disease (primary OTUs) as those OTUs that increased in abundance on corals that ultimately showed disease signs prior to the development of these signs. We assume that corals that were exposed to the infectious dose but did not display disease signs are resistant to the disease (i.e. decrease the pathogen load or prohibit infection) and may therefore not contain OTUs associated with the pathology of the disease within their microbiomes. We therefore focused on OTUs that were more abundant in dosed corals that ultimately displayed disease signs.

We identified OTUs as primary responders if they (i) were absent from the dose; (ii) were present in time-one corals; (iii) differed significantly by final disease state in time-two and three corals; (iv) were more abundant in dosed diseased corals than controls; and (v) did not differ significantly by colony, site or the interaction of site and inoculant.

We identified OTUs as primary colonizers if they (1) were more abundant in the dose than the control inoculant; (ii) differed significantly by final disease state; (iii) were more abundant in dosed diseased corals than control corals at both times two and three; and (iv) did not differ significantly by colony, site and the interaction of inoculant and inoculant site.

Secondary OTUs differed significantly by final disease state and were more abundant in dosed diseased than control corals at time three but not time two. These OTUs were grouped by family, and means were calculated for dosed corals that showed disease signs at time three separated by site and inoculant site.

All sequences were deposited to the Sequence Read Archive under the bioproject ID PRJNA387312. Further specifics of analyses can be found at https:/github.com/sagw/ R-scripts/tree/master/SD1

\section{RESULTS AND DISCUSSION}

We identified groups of OTUs that consistently changed in abundance, contributing to the characteristic diseased coral microbiome: a reduction in resident OTUs associated with certain coral colonies (colony-specific residents), an increase in other resident OTUs (primary responders) and colonization by foreign bacteria (primary colonizers). This method of identifying bacterial groups involved in the transition of a marine animal from health to visible disease signs can be applied to other underexplored marine diseases.

\section{Community-level effects}

Two hundred and seventy-five samples were sequenced yielding 65413553 overlapped reads, which resulted in 97933 OTUs (97\% similarity). The bacterial communities on dosed corals became dramatically more diverse as they developed disease signs in terms of Shannon diversity (from 2.13, SE 0.12 to 4.18 , SE 0.19 , ANOVA, $F_{1,272}=52.37, P<0.001$ ) and rarefied richness (from 224.43 to 402.57 , ANOVA, $F_{1,272}=27.95, P<0.001$ ) (Table S2, Supporting Information). This finding is consistent with other studies of coral disease-associated bacterial communities (e.g. Croquer et al. 2013; Sweet et al. 2013; Gignoux-Wolfsohn and Vollmer 2015; Meyer et al. 2015).

A large amount (18\%) of the variation between bacterial communities of samples collected prior to dosing (at time one) was explained by the significant effect of colony (PERMANOVA, $F_{9,81}$ $\left.=1.8, P=0.001, R^{2}=0.18\right)$. For samples collected after dosing (times two and three), the main effect of final disease state, and the interaction of time, inoculant and inoculant site, significantly affected the coral-associated bacterial communities (Table 1).

\section{Endozoicomonas are colony-specific residents of healthy corals}

In contrast to studies of other species of coral, where Endozoicomonas dominate the microbiomes of all healthy individuals (Yang et al. 2010; Klaus, Janse and Fouke 2011; Apprill, Hughen and Mincer 2013; Bayer et al. 2013; Jessen et al. 2013; Roder et al. 2015, reviewed in Neave et al. 2016a), they were dominant residents of only 4 of the 10 colonies (marked as 'high' in Fig. 1) of healthy Acropora cervicornis, comprising 139 of the 175 OTUs identified as colony-specific residents of healthy corals by GLMM (Table S3, Supporting Information). Endozoicomonas have been shown to form species-specific associations, with different strains found in different species, but they have not previously been shown to vary so drastically among colonies of the same species (Neave et al. 2016b). For these four colonies, Endozoicomonas may be beneficial, since they were less abundant in corals displaying signs of disease than in healthy controls, and were also less abundant in samples of these diseased corals collected at time two prior to visual disease signs (Fig. 2). These Endozoicomonas may only survive in healthy coral tissues; in other species of coral, they have been identified in the endodermal tissues of the host coral (Bayer et al. 2013). Alternatively, Endozoicomonas may be outcompeted by the disease-associated bacteria as the coral contracts disease. Our results suggest that Endozoicomonas may help the coral fight off infection, as they were more abundant in corals that were exposed to the dose but remained healthy than in the healthy controls, which were never exposed to the dose (Fig. 2). A recent study found that removal of Endozoicomonas from the surface mucus layer of corals made corals more susceptible to bleaching and necrosis, highlighting the importance of Endozoicomonas in coral fitness and protecting against foreign bacteria (Glasl, Herndl and Frade 2016). The 


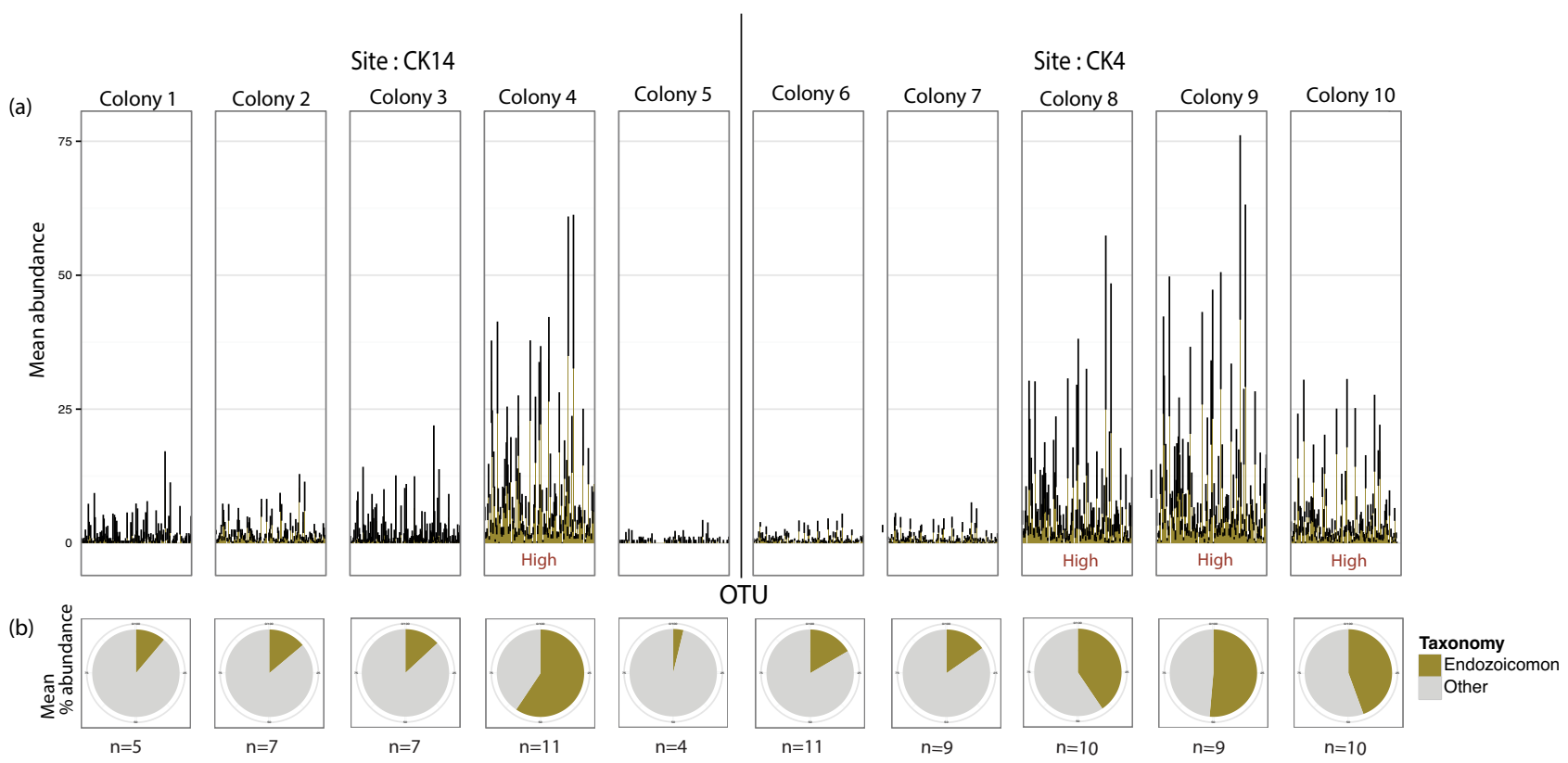

Figure 1. Endozoicomonas are colony-specific resident bacteria of healthy corals. (a) Mean abundance of each resident OTU within each colony at time one; black bars denote standard error. Colonies with greater than $40 \%$ of their total microbiome consisting of Endozoicomonas at time one are labeled 'high'. (b) Percentgae of total microbiome for each colony at time one that is identified as Endozoicomonas or other taxa.

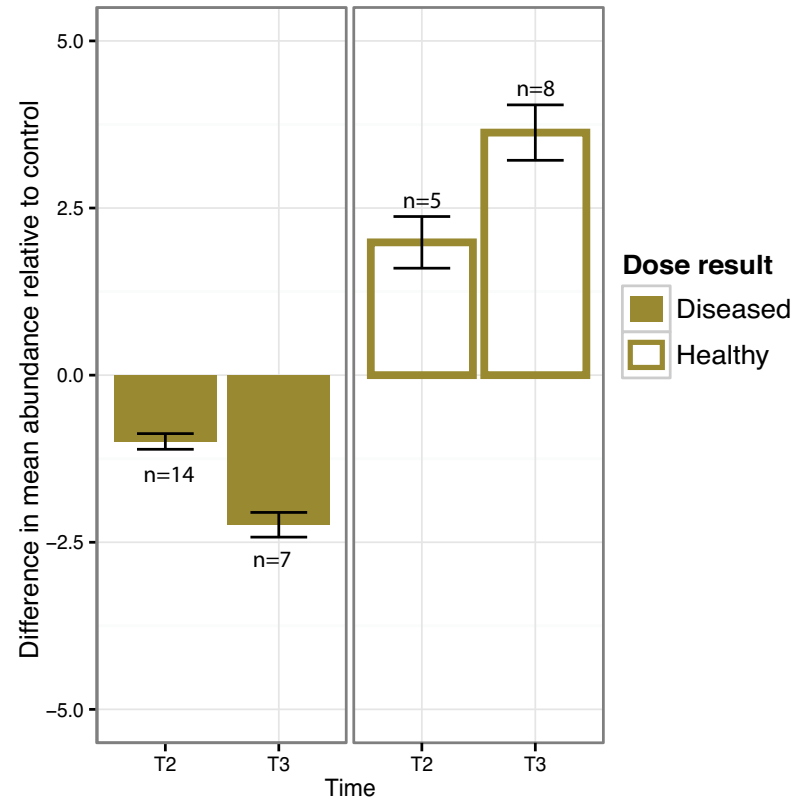

Figure 2. Abundance of resident Endozoicomonas in colonies with greater than $40 \%$ of their total microbiome consisting of Endozoicomonas at time one (high) in dosed corals times two and three. Y-axis is the difference between dosed corals and control corals at each time point; a negative value denotes a lower abundance in dosed corals than controls, and a positive value denotes a higher abundance in dosed corals than controls. Means were calculated for corals exhibiting different final disease states (diseased or healthy) and then control means were subtracted.

observed colony-specificity of Endozoicomonas residents could be due to both the host genetics and the environment. Colonies are likely genetically unique, and a high abundance of Endozoicomonas may be contributing to the disease resistance previously seen in certain genotypes of A. cervicornis (Vollmer and
Kline 2008; Libro and Vollmer 2016). Colonies are also located in slightly different locations on the reef, and Endozoicomonas abundance has been correlated to favorable environmental conditions (Roder et al. 2015). Further investigation into the role Endozoicomonas may be playing in A. cervicornis health and disease resistance will be especially important given the recent finding that Endozoicomonas abundance within A. muricatae tissues decreases with increasing temperatures (Lee et al. 2015).

\section{Secondary OTUs are not consistent across site}

The majority of the 1906 identified secondary OTUs were neither responders (44 OTUs) nor colonizers (222 OTUs). These OTUs were, therefore, presumed to either have originated in the water or been at undetectable abundances when timeone samples were taken. These OTUs appear to contribute the majority of the diversity found in bacterial communities of corals displaying disease signs, but in very low abundances. These low abundances likely contribute to the difficulty in identifying important bacterial groups when comparing the bacterial communities of corals displaying disease signs to those of apparently healthy corals. Contrary to expectations, none of the secondary OTUs were consistent among either site of origin of coral or dose. Rather, all 1906 of these secondary OTUs were also significant for the interaction of 'site', 'inoculant', 'inoculant site' and 'time' (Tables S3 and S4, Supporting Information). These secondary OTUs are unlikely to be involved in development of disease signs, but are more likely attracted to the nutrient source of the dying coral. We only identified secondary OTUs (that were not unique to individual corals or tanks) that increased in abundance when the dose came from the other site (e.g. CK4 corals inoculated with CK14 dose). In the dosed corals that developed disease at time three, 1676 OTUs were more abundant in CK4 corals inoculated with CK14 dose and 230 OTUs were more abundant in CK14 corals inoculated with CK4 dose. Francisellaceae comprised the majority of these secondary OTUs on CK14 corals dosed with 


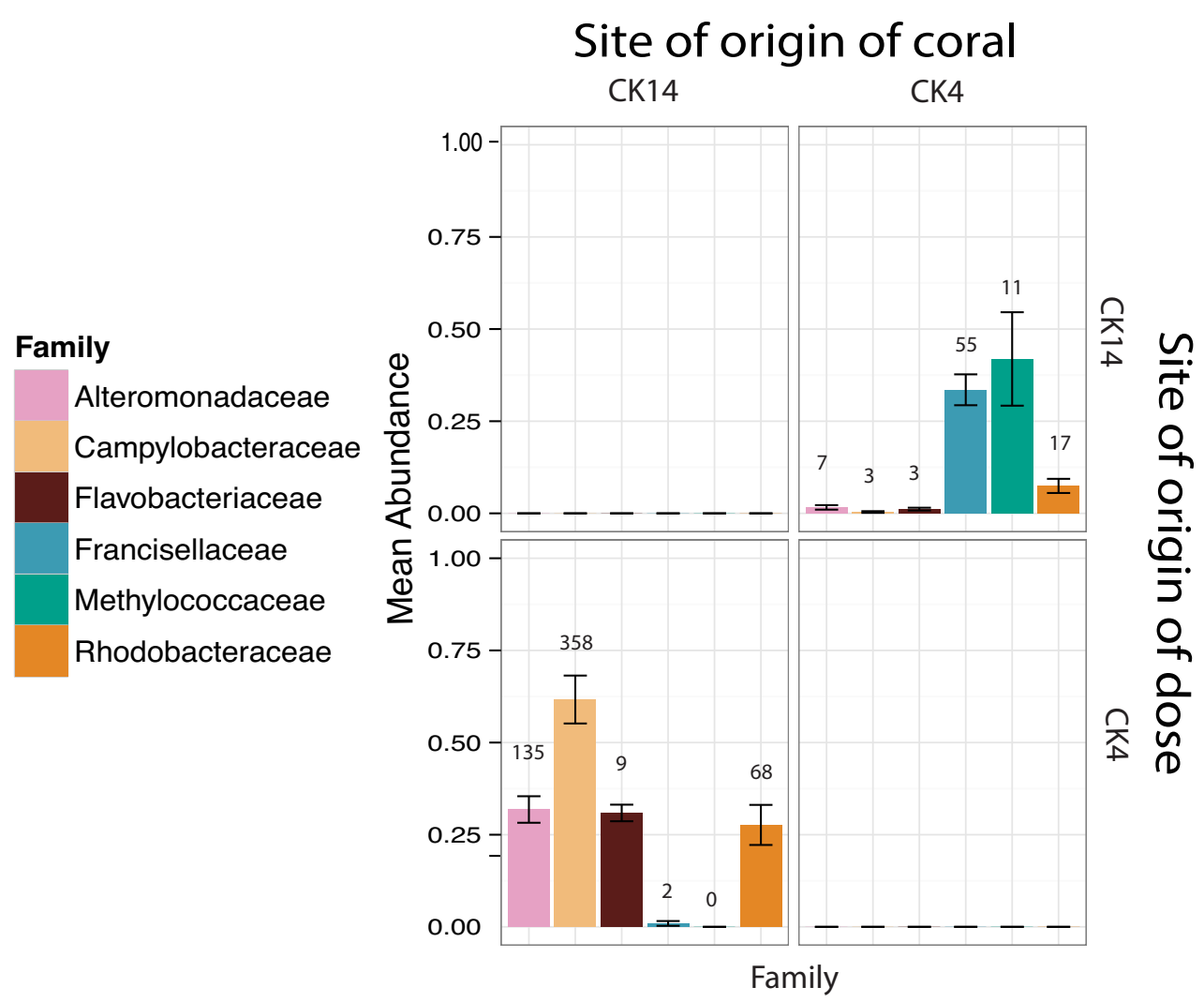

Figure 3. Mean abundance of secondary OTUs belonging to selected families on dosed corals that became diseased at time three. Dosed corals are separated by the site of origin of the dose and the site of origin of the corals. OTUs are grouped by family, and the number of OTUs in each group is noted on the top of the mean abundance bar.

CK4 (55 OTUS), and had the second highest mean abundance after Methylococcaceae. For CK14 corals dosed with CK4, the most abundant family of secondary OTUs with the highest number of OTUs was Campylobacteraceae (358) (Fig. 3). The lack of consistency of secondary OTUs across dose site and site indicates that they are unlikely to be playing a significant role in disease causation. Rather, this pattern suggests that there is an additional secondary disturbance of the bacterial community when the disease-associated bacteria are not taken from surrounding corals.

\section{Primary responders are potential opportunists}

We classified bacteria that were already present in lower numbers on the healthy corals before dosing and responded to the dose by growing more abundant as primary responders. Contrary to our expectations and previous studies suggesting coral disease is caused by opportunistic pathogenesis of resident bacteria (Lesser et al. 2007; Chow, Tang and Mazmanian 2011), all 272 primary responders became more abundant after dosing in all dosed corals regardless of their final disease state (Fig. 4, Table S3). These OTUs, equally abundant in dosed corals that remained healthy and dosed corals that displayed disease signs, are unlikely to be the sole cause of the disease.

Primary responders in the phylum Bacteroidetes, which includes families Flavobacteriaceae (26 OTUs), Cryomorphaceae (22 OTUs) and Saprospiraceae (20 OTUs), appeared to respond to both the disease dose and the general stress of the tank environment by increasing in all corals (including controls) at time three (Fig. 4). We previously found many OTUs belonging to Flavobacteriales (which includes both Flavobacteriaceae and Cryomorphaceae) consistently associated with WBD-infected corals (Gignoux-Wolfsohn and Vollmer 2015). Flavobacteriaceae have been associated with many coral diseases across oceans (Frias-Lopez et al. 2002; Apprill, Hughen and Mincer 2013; Roder et al. 2014b; Ng et al. 2015), cause disease in fish (Starliper 2011) and are part of some healthy marine microbiomes (Apprill et al. 2014). Flavobacteriaceae were recently found to be enriched on algae-dominated reefs, which contain more readily accessible dissolved organic carbon (Haas et al. 2016). Their high abundance in the closed aquaria, which likely grew increasingly nutrient rich as corals died, is consistent with their functioning as copiotrophs; they may be blooming as they consume the dying coral or the secondary metabolites of other members of the diseased bacterial community. We previously identified strains of Saprospiraceae associated with both diseased and healthy corals (Gignoux-Wolfsohn and Vollmer 2015). Members of this family include commonly found marine bacteria involved in the breakdown of complex carbon molecules, consistent with their possible response to a dying or stressed coral (Krieg et al. 2011).

Rather than continuing to increase over time, primary responders belonging to the family Alteromonadaceae (24 OTUs) were most abundant at time two, before any corals displayed disease signs (Fig. 4). These OTUs may grow as an initial response to the introduction of foreign microbes, possibly as defensive symbionts of the host coral. Alteromonadaceae have been previously associated with healthy coral larvae (Ceh, Van Keulen and Bourne 2013) and healthy adult corals (Cardenas et al. 2012), suggesting they can be beneficial symbionts. They are also, however, more abundant in corals infected with multiple diseases (Frias-Lopez et al. 2002; Sunagawa et al. 2009; Roder et al. 2014a,b; Gignoux-Wolfsohn and Vollmer 2015), consistent with a role as 


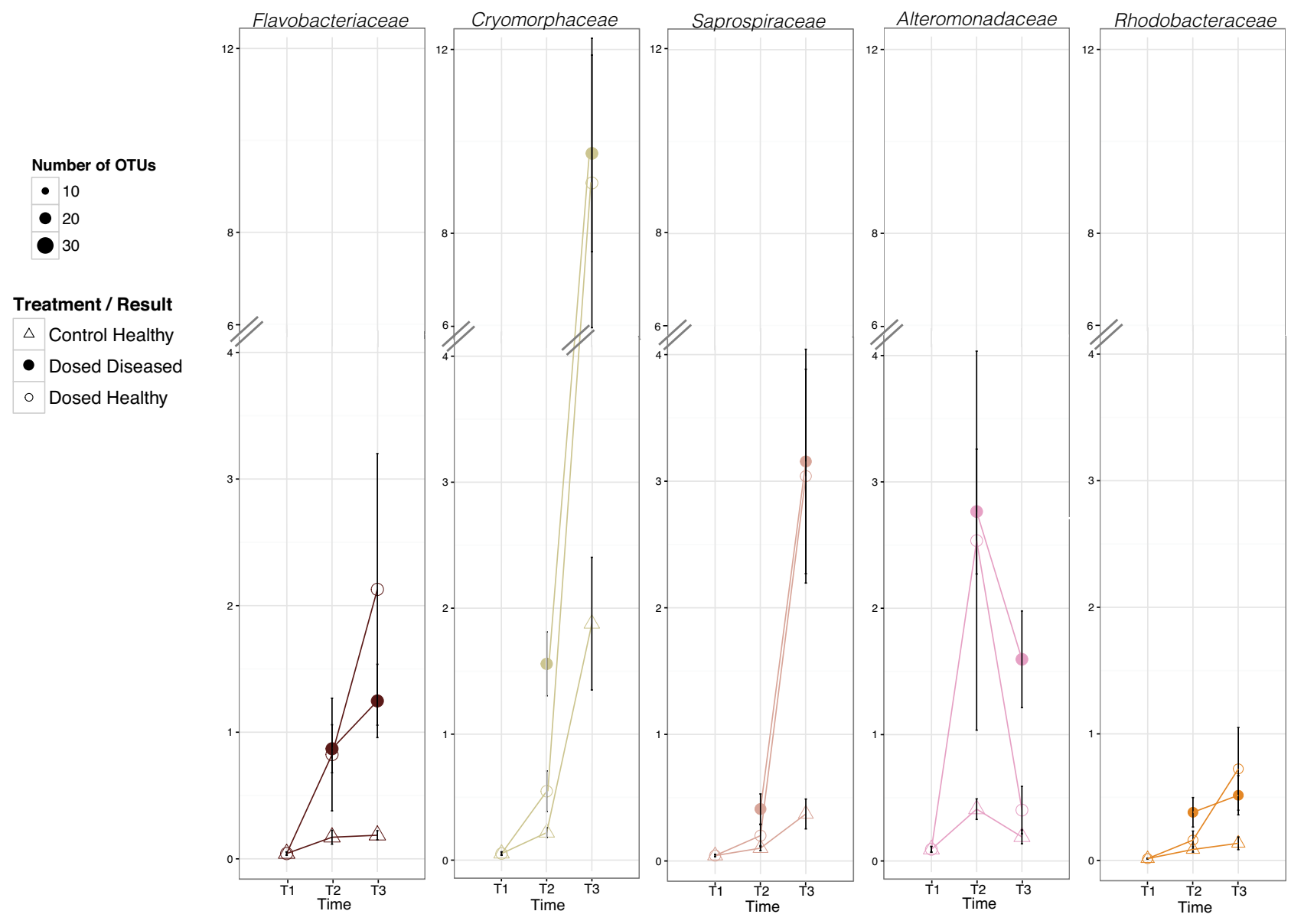

Figure 4. Mean abundance of primary responders belonging to selected families across time. OTUs are grouped by family, and the size of the points denotes how many OTUs belonged to that family.

defensive symbionts. Rhodobacteraceae, the bacterial family most widely associated with coral diseases (summarized in Mouchka, Hewson and Harvell 2010; see also Cardenas et al. 2012; Roder et al. 2014a,b; Gignoux-Wolfsohn and Vollmer 2015; Ng et al. 2015), contained many primary responders (18 OTUs) responding to the dose not the final disease state of the coral (Fig. 4). Rhodobacteraceae seem to be important players in health and disease for multiple coral species (Mouchka, Hewson and Harvell 2010; Glasl, Herndl and Frade 2016), possibly as opportunists or as defensive symbionts, helping the host to fight off infection by foreign bacteria.

\section{Primary colonizers are likely putative primary} pathogens

We identified primary colonizers as 265 OTUs that originated in the disease dose and preferentially colonized corals prior to the development of disease signs, likely after chemotaxing through the water column towards the host coral and attaching to its surface (Fig. 5, Table S3). The coral pathogen Vibrio corallilyticus uses the coral metabolite dimethylsulfoniopropionate to locate potential hosts (Garren et al. 2014); it is possible that the pathogen(s) may use a similar method of host location. It is interesting, therefore, that we did not only identify one species of bacteria that originated in the dose and colonized corals prior to disease signs, but rather many sometimes distantly related OTUs. This result likely explains the difficulty in identifying primary pathogens of coral diseases and indicates that there may not be a single primary pathogen, but a consortium of bacteria that cause disease signs. Evidence that quorum sensing is important in contraction of WBD provides a possible method for interspecies communication and infection by a consortium (Certner and Vollmer 2015). The previously suggested WBD pathogens, Vibrionaceae (one OTU) and Rickettsiaceae (five OTUs) were not more abundant in dosed corals that displayed disease signs than those that remained healthy, making them unlikely primary pathogens in this experiment (Fig. 5).

Interestingly, taxonomy did not always dictate where and when OTUs were found. Many primary colonizers and primary responders were identified as belonging to the families Flavobacteriaceae and Alteromonadaceae. The 22 Flavobacteriaceae identified as colonizers again appear to be acting as copiotrophs, and may have been abundant in the dose because they were primary responders on the corals used to create the dose. In contrast, the 22 Alteromonadaceae identified as primary colonizers followed a different pattern from the Alteromonadaceae OTUs in the primary responders group. Instead, their pattern of abundance was similar to primary colonizer OTUs belonging to other families including Campylobacteraceae (25 OTUs), Francisellaceae (38 OTUs) and Pasteurellaceae (26 OTUs) -only colonizing corals prior to the development of disease signs and proliferating as the disease progressed (Fig. 5).

The absence of many groups of primary colonizers from corals that were dosed but did not display disease signs indicates 


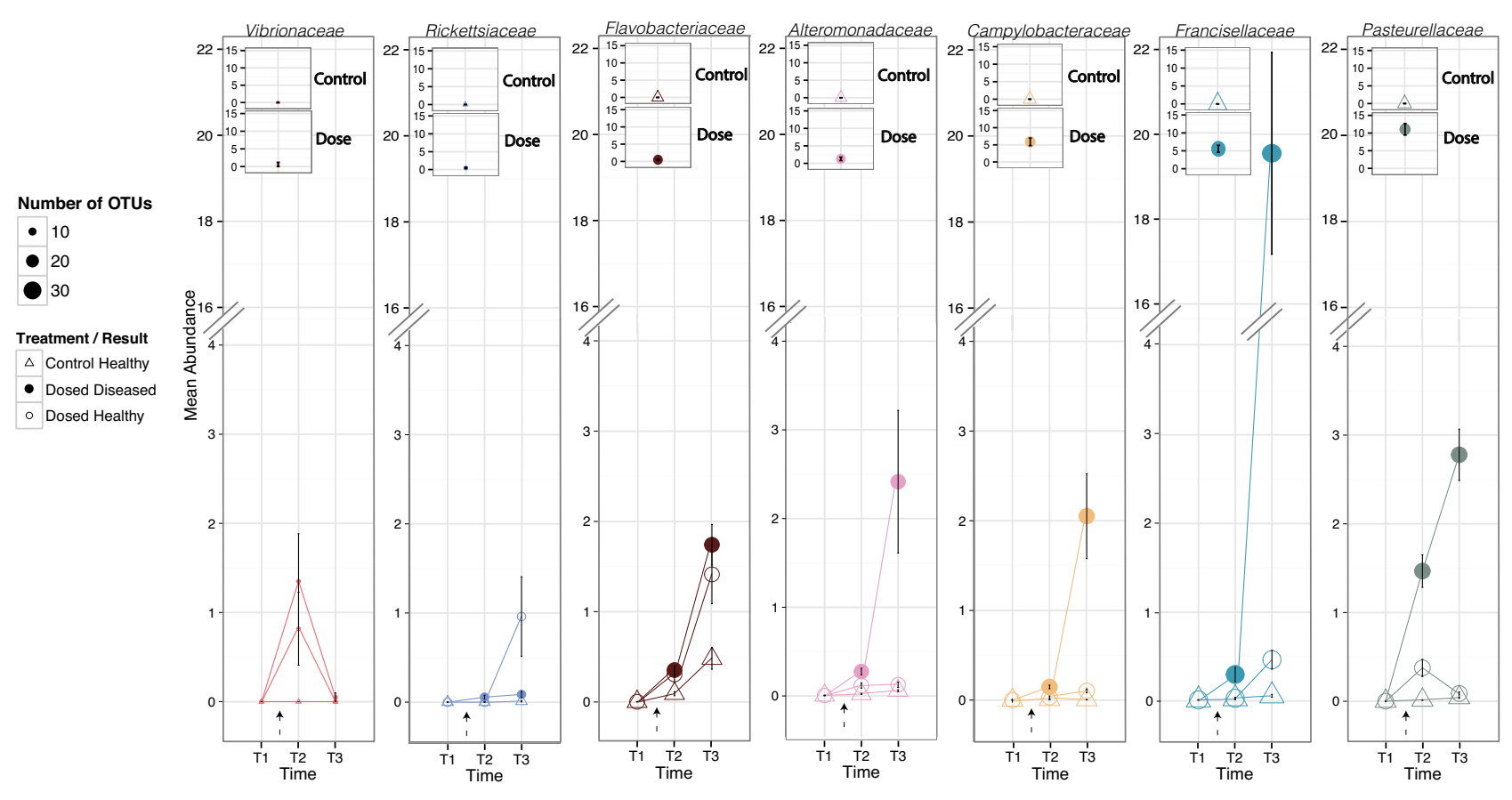

Figure 5. Mean abundance of primary colonizers belonging to selected families across time. OTUs are grouped by family, and the size of the points denotes how many OTUs belonged to the specified family. Inset is the mean abundance for OTUs in that family in the inoculants (dose and control). Arrows signify time of inoculation. Error bars denote standard error.

that these OTUs are likely directly involved in the development of WBD signs (Fig. 5). Members of the Campylobacteraceae family have been associated with multiple coral diseases including WBD (e.g. Sunagawa et al. 2009; Sweet and Bythell 2012; Sweet et al. 2013; Roder et al. 2014a; Gignoux-Wolfsohn and Vollmer 2015). In other systems, Campylobacteraceae are known to be both commensal and zoonotic pathogens (Stoddard et al. 2005; Lee and Newell 2006). In contrast, Francisellaceae have not been previously associated with coral disease, but are common marine bacteria (Duodu et al. 2012), which can be intracellular pathogens of both Atlantic cod (Wangen et al. 2012) and humans (Sjostedt 2006) and are also endosymbionts of ciliates (Schrallhammer et al. 2011).

Primary colonizers in the family Pasteurellaceae exhibited a pattern of colonization consistent with a strong involvement in disease: these OTUs were very abundant in the dose and preferentially colonized dosed corals before they showed disease signs with a more dramatic increase in abundance than any other family (Fig. 5). Pasteurellaceae have not been previously associated with coral disease, but they are common pathogens of many other animals including humans (Johnson and Rumans 1977; Frey and Kuhnert 2002) and were recently found to be enriched on reefs with high algal cover (Haas et al. 2016). One possible explanation for our identification of Pasteurellaceae, and not $\mathrm{V}$. charchariae behaving like a primary WBD pathogen, is that Pasteurellaceae may be an emerging pathogen of Panamanian corals that also causes WBD-like signs. The increasingly algae-dominated Panamanian reefs may promote new coral pathogens that cause macroscopic signs similar to canonical WBD.

While we identified some consistent actors in the diseased coral microbiome, we did not explain the majority of variation between samples, indicating there are other factors not examined in this study that shape the coral microbiome. This study used corals displaying disease signs consistent with WBD from Panama; whether the patterns described here apply to all corals displaying WBD-like signs across the Caribbean is unknown. We were limited by the length of the region sequenced and the available databases-as technology and resources improve, bacterial taxonomy will be better resolved.

\section{CONCLUSIONS}

The diseased coral microbiome is dependent on the pre-existing healthy microbiome, the disease history of the infected coral, the origin of the disease and the timing of disease progression. Our approach allowed us to separate bacteria based on origin and timing of increased abundance, providing more information than previous culture-independent studies about what bacteria are likely contributing to disease. Our finding that Endozoicomonas are only associated with health on certain coral colonies may explain the variation in responses of individual corals to disease. The discovery that primary responders, likely opportunists, increase in dosed corals regardless of final disease state negates hypotheses that WBD on Acropora cervicornis is not caused solely by opportunists. We identified primary colonizers originating in the infectious dose and were able to closely track their changes in abundance as corals developed disease signs, identifying Campylobacteraceae, Francisellaceae and Pasteurellaceae as the most likely primary pathogens. Our results underscore the importance of incorporating time into future studies of marine diseases and the need to observe the behavior of individual bacterial strains rather than summarizing changes in communities only by higher-level taxonomy. Our approach can be applied to other marine diseases that do not fit into a one-pathogen onedisease framework, providing a more holistic understanding of disease and allowing for the shifting definitions of pathogens within our changing marine climate.

\section{SUPPLEMENTARY DATA}

Supplementary data are available at FEMSEC online. 


\section{ACKNOWLEDGEMENTS}

We thank H. Nelson for help with sample collection; T. Gouhier for help with statistical analyses; E. Holum for help with Bioinformatics; M. Garren, J. Grabowski, E. Weil, R. Certner and S. Kopac for comments on the manuscript.

\section{FUNDING}

This work was supported by a crowd-funding campaign to SGW and FA: https://experiment.com/projects/what-iskilling-caribbean-corals-investigating-a-devastating-coral-disease and National Science Foundation funding to SV (NSF OCE-1 458 158). Collection permit was provided by Autoridad Nacional del Ambiente (ANAM): SE/A-108-13.

Conflict of interest. None declared.

\section{REFERENCES}

Apprill A, Hughen K, Mincer T. Major similarities in the bacterial communities associated with lesioned and healthy Fungiidae corals. Environ Microbiol 2013;15:2063-72.

Apprill A, Robbins J, Eren AM et al. Humpback whale populations share a core skin bacterial community: towards a health index for marine mammals? PLoS One 2014;9:e90785.

Aronson RB, Precht WF. White-band disease and the changing face of Caribbean coral reefs. Hydrobiologia 2001;460:25-38.

Barriuso J, Valverde JR, Mellado RP. Estimation of bacterial diversity using next generation sequencing of $16 \mathrm{~S}$ rDNA a comparison of different workflows. BMC Bioinformatics 2011;12:473.

Bayer T, Neave MJ, Alsheikh-Hussain A et al. The microbiome of the Red Sea Coral Stylophora pistillata is dominated by tissue-associated Endozoicomonas bacteria. Appl Environ Microb 2013;79:4759-62.

Burge CA, Mark Eakin C, Friedman CS et al. Climate change influences on marine infectious diseases: implications for management and society. Annu Rev Mar Sci 2014;6:249-77.

Caporaso JG, Lauber CL, Walters WA et al. Ultra-high-throughput microbial community analysis on the Illumina HiSeq and MiSeq platforms. ISME J 2012;6:1621-4.

Cardenas A, Rodruiguez-R LM, Pizarro V et al. Shifts in bacterial communities of two caribbean reef-building coral species affected by white plague disease. ISME J 2012;6:502-12.

Casas V, Kline DI, Wegley L et al. Widespread association of a Rickettsiales-like bacterium with reef-building corals. Environ Microbiol 2004;6:1137-48.

Cavanaugh CM, Gardiner SL, Jones ML et al. Prokaryotic cells in the hydrothermal vent tube worm Riftia pachyptila jones: possible chemoautotrophic symbionts. Science 1981;213:340-2.

Ceh J, Van Keulen M, Bourne DG. Intergenerational transfer of specific bacteria in corals and possible implications for offspring fitness. Microb Ecol 2013;65:227-31.

Certner RH, Vollmer SV. Evidence for autoinduction and quorum sensing in white band disease-causing microbes on Acropora cervicornis. Sci Rep 2015;5:11134.

Chow J, Tang H, Mazmanian SK. Pathobionts of the gastrointestinal microbiota and inflammatory disease. Curr Opin Immunol 2011;23:473-80.

Closek CJ, Sunagawa S, Desalvo MK et al. Coral transcriptome and bacterial community profiles reveal distinct Yellow Band Disease states in Orbicella faveolata. ISME J 2014;8:2411-22.

Croquer A, Bastidas C, Elliott A et al. Bacterial assemblages shifts from healthy to yellow band disease states in the dominant reef coral Montastraea faveolata. Environ Microbiol Rep 2013;5:90-6.
Duodu S, Larsson P, Sjodin A et al. The distribution of Francisellalike bacteria associated with coastal waters in Norway. Microb Ecol 2012;64:370-7.

Edgar RC, Haas BJ, Clemente JC et al. UCHIME improves sensitivity and speed of chimera detection. Bioinformatics 2011;27:2194-200.

Fox J, Weisberg S. An $\{R\}$ Companion to Applied Regression, 2nd edn. Thousand Oaks, CA: Sage, 2011.

Frey J, Kuhnert P. RTX toxins in Pasteurellaceae. Int J Med Microbiol 2002;292:149-58.

Frias-Lopez J, Zerkle AL, Bonheyo GT et al. Partitioning of bacterial communities between seawater and healthy, black band diseased, and dead coral surfaces. Appl Environ Microb 2002;68:2214-28.

Fukami H, Budd AF, Levitan DR et al. Geographic differences in species boundaries among members of the Montatstraea Annularis complex based on molecular and morphological markers. Evolution 2004;58:324-37.

Garren M, Azam F. Corals shed bacteria as a potential mechanism of resilience to organic matter enrichment. ISME J 2012;6:1159-65.

Garren M, Son K, Raina JB et al. A bacterial pathogen uses dimethylsulfonioproprionate as a cue to target heat-stressed corals. ISME J 2014;8:999-1007.

Gignoux-Wolfsohn SA, Marks CJ, Vollmer SV. White Band Disease transmission in the threatened coral, Acropora cervicornis. Sci Rep 2012;2:804.

Gignoux-Wolfsohn SA, Vollmer SV. Identification of candidate coral pathogens on white band disease-infected staghorn coral. PLoS One 2015;10:e0134416.

Gil-Agudelo DL, Smith GW, Weil E. The white band disease type II pathogen in Puerto Rico. Rev Biol Trop 2006;54:59-67.

Gladfelter WB, Gladfelter EH, Monahan RK et al. Environmental studies of buck island reef national monument. The National Park Service U.S. Department of Interior 1977.

Gladfelter WB. White-band disease in Acropora palmata: implications for the structure and growth of shallow reefs. Bull Mar Sci 1982;32:639-43.

Glasl B, Herndl GJ, Frade PR. The microbiome of coral surface mucus has a key role in mediating holobiont health and survival upon disturbance. ISME J 2016;10:2280-92.

Gloor GB, Hummelen R, Macklaim JM et al. Microbiome profiling by illumina sequencing of combinatorial sequence-tagged PCR products. PLoS One 2010;5:e15406.

Haas AF, Fairoz MFM, Kelly LW et al. Global microbialization of coral reefs. Nat Microbiol 2016;1:16042.

Jessen C, Villa Lizcano JF, Bayer T et al. In-situ effects of eutrophication and overfishing on physiology and bacterial diversity of the red sea coral Acropora hemprichii. PLoS One 2013;8:e62091.

Johnson RH, Rumans LW. Unusual infectious caused by Pasteurella multocida. J Am Med Assoc 1977;237:146-7.

Klaus JS, Janse I, Fouke BW. Coral black band disease microbial communities and genotypic variability of the dominant cyanobacteria (CD1C11). Bull Mar Sci 2011;87:795821.

Kline DI, Vollmer SV. White band disease (Type I) of endangered caribbean acroporid corals is caused by pathogenic bacteria. Sci Rep 2011;1:7.

Krieg N, Staley J, Brown D et al. Family III. Saprospiraceae fam. nov. In: Krieg NR, Staley JT, Brown DR et al. (eds). Bergey's Manual of Systematic Bacteriology. New York: Springer, 2011, 358-70.

Lee M, Newell D. Invited minireview: Campylobacter in poultry: filling an ecological niche. Avian Dis 2006;50:1-9. 
Lee ST, Davy SK, Tang SL et al. Successive shifts in the microbial community of the surface mucus layer and tissues of the coral Acropora muricata under thermal stress. FEMS microbiology ecology 2015;91.

Lemire A, Goudenege D, Versigny T et al. Populations, not clones, are the unit of vibrio pathogenesis in naturally infected oysters. ISME J 2015;9:1523-31.

Lesser MP, Bythell JC, Gates RD et al. Are infectious diseases really killing corals? Alternative interpretations of the experimental and ecological data. J Exp Mar Biol Ecol 2007;346:36-44.

Libro S, Vollmer SV. Genetic signature of resistance to white band disease in the caribbean staghorn coral Acropora cervicornis. PLoS One 2016;11:e0146636.

Love MI, Huber W, Anders S. Moderated estimation of fold change and dispersion for RNA-seq data with DESeq2. Genome Biol 2014;15:550.

Mcmurdie PJ, Holmes S. Waste not, want not: Why rarefying microbiome data is Inadmissible. PLos Comput Biol 2014;10:e1003531.

Magoc T, Salzberg SL. FLASH: Fast length adjustment of short reads to improve genome assemblies. Bioinformatics 2011;27:2957-63.

Meyer JL, Gunasekera SP, Scott RM et al. Microbiome shifts and the inhibition of quorum sensing by Black Band Disease cyanobacteria. ISME J 2015;10:1204-16.

Mouchka ME, Hewson I, Harvell CD. Coral-associated bacterial assemblages: current knowledge and the potential for climate-driven impacts. Integr Comp Biol 2010;50:662-74.

Neave MJ, Apprill A, Ferrier-Pages C et al. Diversity and function of prevalent symbiotic marine bacteria in the genus Endozoicomonas. Appl Microbiol Biot 2016a;100:8315-24.

Neave MJ, Rachmawati R, Xun L et al. Differential specificity between closely related corals and abundant Endozoicomonas endosymbionts across global scales. ISME J 2016b;11:186-200.

Ng JC, Chan Y, Tun HM et al. Pyrosequencing of the bacteria associated with Platygyra carnosus corals with skeletal growth anomalies reveals differences in bacterial community composition in apparently healthy and diseased tissues. Front Microbiol 2015;6:1142.

Nyholm SV, Mcfall-Ngai MJ. The winnowing: establishing the squid-vibrio symbiosis. Nat Reu Microbiol 2004;2:632-42.

Oksanen J, Guillaume Blanchet F, Kindt R et al. Vegan: Community Ecology Package. R package version 2.0-9. 2013.

Peters EC, Oprandy J, Yevich P. Possible causal agent of "White Band Disease" in Caribbean Acroporid Corals. J Invertebr Pathol 1983;41:394-6.

Quast C, Pruesse E, Yilmaz P et al. The SILVA ribosomal RNA gene database project: improved data processing and web-based tools. Nucleic Acids Res 2013;41:D590-6.

Randall CJ, van Woesik R. Contemporary white-band disease in Caribbean corals driven by climate change. Nat Clim Chang 2015.

Roder C, Arif C, Bayer T et al. Bacterial profiling of White Plague Disease in a comparative coral species framework. ISME $J$ 2014a;8:31-9.

Roder C, Arif C, Daniels C et al. Bacterial profiling of White Plague Disease across corals and oceans indicates a conserved and distinct disease microbiome. Mol Ecol 2014b;23:965-74.

Roder C, Bayer T, Aranda M et al. Microbiome structure of the fungid coral Ctenactis echinata aligns with environmental differences. Mol Ecol 2015;24:3501-11.

Rutherford ST, Bassler BL. Bacterial quorum sensing: its role in virulence and possibilities for its control. Cold Spring Harb Perspect Med 2012;2.
Sachs JL, Skophammer RG, Regus JU. Evolutionary transitions in bacterial symbiosis. PNAS 2011;108:10800-7.

Sayavedra L, Kleiner M, Ponnudurai R et al. Abundant toxinrelated genes in the genomes of beneficial symbionts from deep-sea hydrothermal vent mussels. Elife 2015;4:e07966.

Schrallhammer M, Schweikert M, Vallesi A et al. Detection of a novel subspecies of Francisella noatunensis as endosymbiont of the ciliate Euplotes raikovi. Microb Ecol 2011;61:455-64.

Sjostedt A. Intracellular survival mechanisms of Francisella tularensis, a stealth pathogen. Microbes Infect 2006;8:561-7.

Starliper CE. Bacterial coldwater disease of fishes caused by Flavobacterium psychrophilum. J Adv Res 2011;2 97-108.

Stoddard R, Gulland F, Atwill E et al. Salmonella and Campylobacter spp. in Northern Elephant Seals, California. Emerg Infect Dis 2005;11:1967-9.

Sunagawa S, Desantis TZ, Piceno YM et al. Bacterial diversity and white plague disease-associated community changes in the Caribbean coral Montastraea faveolata. ISME J 2009;3:51221.

Sweet M, Bythell J. Ciliate and bacterial communities associated with White Syndrome and Brown Band Disease in reefbuilding corals. Environ Microbiol 2012;14:2184-99.

Sweet M, Burn D, Croquer A et al. Characterisation of the bacterial and fungal communities associated with different lesion sizes of dark spot syndrome occurring in the coral Stephanocoenia interstepta. PLoS One 2013;8:e62580.

Sweet MJ, Croquer A, Bythell JC. Experimental antibiotic treatment identifies potential pathogens of white band disease in the endangered Caribbean coral Acropora cervicornis. Proc Biol Sci 2014;281. DOI: 10.1098/rspb.2014.0094.

Tout J, Siboni N, Messer LF et al. Increased seawater temperature increases the abundance and alters the structure of natural Vibrio populations associated with the coral Pocillopora damicornis. Front Microbiol 2015;6:432.

Tunnicliffe V. Caribbean staghorn coral populations: preHurricane Allen conditions in Discovery Bay, Jamaica. Bull Mar Sci 1983;33:132-51.

Venables W, Ripley B. Modern Applied Statistics with S, 4th edn. New York: Springer, 2003.

Vollmer SV, Kline DI. Natural disease resistance in threatened staghorn corals. PLoS One 2008;3:e3718.

Wangen IH, Karlsbakk E, Einen AC et al. Fate of Francisella noatunensis, a pathogen of Atlantic cod Gadus morhua, in blue mussels Mytilus edulis. Dis Aquat Organ 2012;98:63-72.

Weil E. Coral reef diseases in the wider Caribbean. In: Coral Health and Disease. Berlin, Heidelberg: Springer, 2004, 35-68.

Weil E, Rogers CS. Coral Reef Diseases in the Atlantic-Caribbean. Netherlands: Springer, 2011, 465-91.

Wickham H. ggplot2: Elegant Graphics for Data Analysis. New York: Springer Science \& Business Media. 2009.

Yang CS, Chen MH, Arun AB et al. Endozoicomonas montiporae sp. nov., isolated from the encrusting pore coral Montipora aequituberculata. Int J Syst Evol Microbiol 2010;60:1158-62.

Youssef N, Sheik CS, Krumholz LR et al. Comparison of species richness estimates obtained using nearly complete fragments and simulated pyrosequencing-generated fragments in 16S rRNA gene-based environmental surveys. Appl Environ Microb 2009;75:5227-36.

Zaneveld JR, Burkepile DE, Shantz AA et al. Overfishing and nutrient pollution interact with temperature to disrupt coral reefs down to microbial scales. Nat Commun 2016;7:11833.

Zwart MP, Daros JA, Elena SF. One is enough: in vivo effective population size is dose-dependent for a plant RNA virus. PLoS pathogens 2011;7:e1002122. 